\title{
Waveguide Fabry-Pérot microcavity arrays
}

\author{
Shengfei Feng, ${ }^{1,2}$ Xinping Zhang, ${ }^{1, a)}$ and Peter J. Klar ${ }^{2}$ \\ ${ }^{1}$ Institute of Information Photonics Technology and College of Applied Sciences, Beijing University \\ of Technology, Beijing 100124, People's Republic of China \\ ${ }^{2}$ Institute of Experimental Physics I, Justus-Liebig-University Giessen, Germany
}

(Received 14 March 2011; accepted 30 June 2011; published online 4 August 2011)

\begin{abstract}
Spectroscopic properties of the Fabry-Pérot (FP) microcavities arranged periodically on a dielectric waveguide are investigated theoretically. A strong coupling behavior between the waveguide resonance and the FP resonance modes is demonstrated for incident light with TE polarization. The narrow-band waveguide resonance modes are observed as asymmetric transmission peaks within the FP stop band, whereas they become transmission dips (antiresonance) in the FP pass band. The coupled mode transits between these two states as the waveguide resonance mode is tuned between the FP pass and stop bands. This transition can be explained in the Fano-picture and is important for the design of photonic devices with narrow-band and tunable optical response (C) 2011 American Institute of Physics. [doi:10.1063/1.3616148]
\end{abstract}

Interactions between different resonance modes in photonic structures may introduce coupled modes that can be utilized to explore special optical functions that cannot be realized by conventional methods. For example, sharp peaks in the reflection and dips in the transmission spectra have been observed in waveguide grating structures, ${ }^{1}$ which might be used to develop narrow-band filters, ${ }^{2}$ polarizers, ${ }^{3}$ or optical switches. ${ }^{4}$ Anti-crossing interaction behavior has been observed in waveguided metallic photonic crystals (MPCs) as a result of the coupling between the narrow-band waveguide resonance mode and the broad-band particle plasmon resonance of the metallic nanostructures. ${ }^{5}$ In these cases, the lineshape of the narrow-band resonance can be well described in the Fano picture. ${ }^{6}$ The behavior of these narrow-band coupled modes needs to be understood to employ them in sensitive sensors. ${ }^{7}$

In this paper, we demonstrate theoretically the special optical response of a nano-optical device consisting of an array of Fabry-Pérot (FP) microcavities periodically arranged on a dielectric waveguide, as shown schematically in Fig. 1. All the FP microcavities consist of a thin layer of $\mathrm{ZnS}$ sandwiched between two silver slabs. ZnS is a II-VI semiconductor with high transparency in the visible spectral range and a high refractive index $\left(n_{\mathrm{ZnS}}=2.30-2.57\right)$, which makes it suitable for a waveguide medium. Furthermore, $\mathrm{ZnS}$ can be deposited easily using a variety of techniques. ${ }^{8}$ Silver is employed because it is superior to gold and other metals in terms of a higher and flatter reflection spectrum and relatively lower absorption in the visible spectral range. The periodical FP microcavity array has a period of $\Lambda$ and a modulation depth of $d+2 t$, where $t$ is the thickness of the silver slabs and $d$ is the thickness of the $\mathrm{ZnS}$ layer. The duty cycle of the grating structures is defined as $f=w / \Lambda$, where $w$ is the width of the FP microcavity. The waveguide that is also made of $\mathrm{ZnS}$ has a thickness of $h$. The substrate is assumed to be made of glass with a refractive index of $n_{\text {sub }}=1.5$.

Periodical arrangement of the FP microcavities leads to a grating structure that diffracts the incident light to excite the

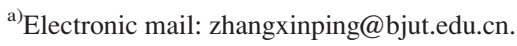

propagation mode in the waveguide. Thus, two resonance modes can be observed in the transmission: the narrow-band waveguide resonance mode and the relatively broad band FP microcavity resonance mode. In the waveguide grating structures, a ray picture model ${ }^{1}$ was established to calculate the optical electric field of the transmitted light wave $(E)$ with $E=\left(S_{t}+S_{W}\right) E_{0}$, where $E_{0}$ is the incident optical electric field. The term $S_{t}$ denotes the direct transmission coefficient of the optical electric field, and $S_{W}$ results from the sum of the diffractions of the waveguide propagation mode by the grating with

$$
S_{W}=\exp (-i \pi) \frac{S \exp (i \Delta)}{1-(1-S) \exp (i \Delta)},
$$

where $S$ is related to the diffraction efficiency of the grating and $\Delta$ is the phase-mismatch from the resonant condition $(\Delta=0)$ and is as defined in Ref. 9.

For the waveguide Fabry-Pérot microcavities studied here, the directly transmitted optical electric field is modulated by the FP resonance with a coefficient of $S_{F P}$, so that

$$
S_{t}=S_{F P}=\eta_{\text {eff }} \frac{1-R_{\text {eff }}}{1-R_{\text {eff }} \exp \left(i \frac{2 \pi n_{Z n s} d_{e f f}}{\lambda}\right)},
$$

where the coefficient $\eta_{\text {eff }}$ is introduced due to the space filling factor of the FP microcavities over the whole grating area, which is dependent on the duty cycle of the grating

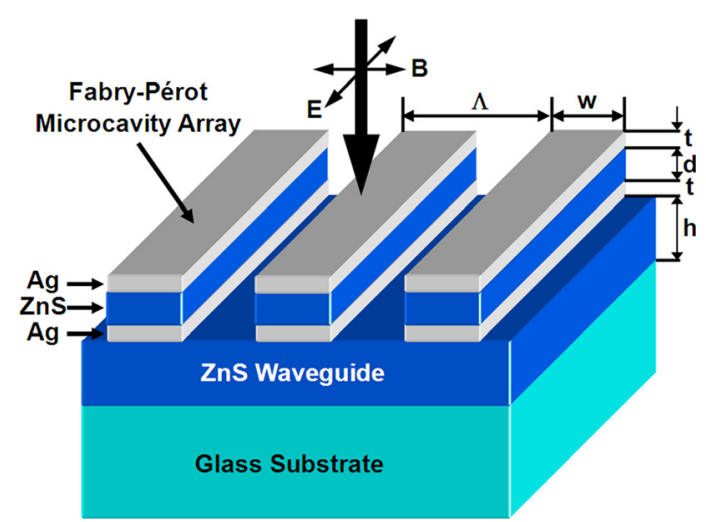

FIG. 1. (Color online) Schematic illustration of the design of the waveguide FP microcavity array. 


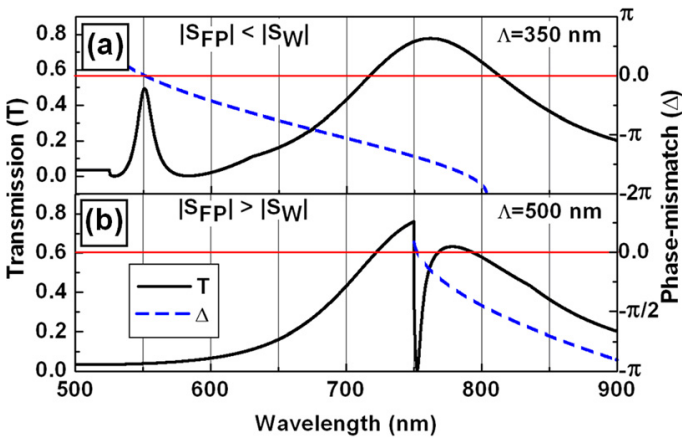

FIG. 2. (Color online) Transmission spectra ( $T$ ) of the waveguide FP microcavity arrays calculated by the modified ray picture model for (a) $\Lambda=350$ $\mathrm{nm}$ with the waveguide resonant mode overlap the FP stop band and $\left|S_{F P}\right|<\left|S_{W}\right|$, (b) $\Lambda=500 \mathrm{~nm}$ with the waveguide resonance mode overlap the FP pass band and $\left|S_{F P}\right|>\left|S_{W}\right|$. The corresponding phase mismatch $(\Delta)$ is also plotted as a function of wavelength. Other parameters used in the calculation: $R_{\text {eff }}=0.5, \eta_{\text {eff }}=0.8, d_{\text {eff }}=166 \mathrm{~nm}, n_{\mathrm{ZnS}}=2.32, S=0.2, n_{\text {sub }}=1.5$, and $h=75 \mathrm{~nm}$.

structures, $R_{\text {eff }}$ is the effective reflection coefficient of the silver slabs, $d_{\text {eff }}$ is the effective cavity length, and $n_{\mathrm{ZnS}}$ is the refractive index of the $\mathrm{ZnS}$ layer at a wavelength of $\lambda$. Thus, the transmissivity through the waveguide Fabry-Perot microcavity arrays may be written as $T=\left|E / E_{0}\right|^{2}=\left|S_{t}+S_{W}\right|^{2}$.

Therefore, interference may take place between the directly transmitted and the diffracted light beams. Such a situation was originally described by Fano. ${ }^{10}$ Light in the spectral region of the narrow-band waveguide resonance mode has two possible paths to propagate through the structure, which can be either by the waveguide mode or by the quasi-continuous FP resonance mode. The coupling between them leads to the interference effects, which are also manifested in the lineshape of the waveguide resonance mode described by the so called Fano-lineshape $\mathrm{F}(\varepsilon)=(\varepsilon+q)^{2} /\left(\varepsilon^{2}+1\right)$, where $\varepsilon=2\left(E-E_{\mathrm{wg}}\right) /$ $\Gamma . E_{\mathrm{wg}}$ and $\Gamma$ are the energy and the linewidth of the waveguide resonance, $q$ is the Fano-parameter given by the ratio of the probabilities of light passing through the waveguide resonance and through the corresponding FP resonance modes.

The resonance of FP microcavities introduces pass bands and leaves stop bands in the transmission spectrum (Fig. 2). The diffraction of the propagation mode in the waveguide by the top grating produces multiple beams that propagate in the same direction as the directly transmitted beam. If the diffracted light is much stronger than the direct transmission $\left(\left|S_{F P}\right|<\left|S_{w}\right|\right)$, corresponding to the spectral overlap between the waveguide resonance mode and the FP stop band, destructive interference between them leaves a symmetric peak in the transmission spectrum $(q=0)$. However, if the direct transmission dominates $\left(\left|S_{F P}\right|>\left|S_{w}\right|\right)$ in regions where the waveguide resonance mode overlaps the pass band of the FP microcavities, the destructive interference between them produces a dip or antiresonance in the transmission spectrum $(q=\infty)$. In the intermediate range, the lineshape of the waveguide resonance mode is asymmetric (finite $q$ ). These effects depend on the diffraction efficiency by the grating of FP microcavities, the duty cycle of the grating, and the strength of the FP resonance mode. The phase-mismatch $(\Delta)$ is shown as a function of wavelength by the dashed curves in Fig. 2. " $\Delta=0$ " takes place at 550 and $752 \mathrm{~nm}$ for a grating period of $350 \mathrm{~nm}$ (Fig. 2(a)) and 500 nm (Fig. 2(b)), respectively. However, the phase-mismatch cannot be evaluated for wavelengths shorter than $750 \mathrm{~nm}$ due to the unsatisfied total reflection condition and a dramatic falling edge is observed at $750 \mathrm{~nm}$. In the calculations in Fig. 2, the effective reflectivity $R_{\text {eff }}$ was reasonably set to 0.5 for a silver film as thick as $25 \mathrm{~nm}, S$ is set to 0.2 for a duty cycle of about 0.5 and a modulation depth of $<200 \mathrm{~nm}$, The thickness of the silver film $(t=25 \mathrm{~nm})$ has been chosen to make the silver film that has a reasonable reflectivity and the FP cavity that has a reasonable transmission spectrum. The effective FP cavity length $\left(d_{e f f}\right)$ is adjusted to about 166 $\mathrm{nm}$ so that two clearly different coupling states can be observed within the visible. $\eta_{\text {eff }}$ characterizes the modulation of the FP resonance on the transmission of the light and is set to 0.8 on the basis of the simulation results using a duty cycle of 0.5 .

The software of GSOLVER (Ref. 11) based on the full vector implementation of the algorithms of the so-called rigorous coupled wave analysis ${ }^{12}$ was employed to simulate the transmission spectra of the structure. For simplicity, the incident light is assumed to be polarized parallel to the extending direction of the "grating" (TE polarization) and was incident parallel to the normal of the substrate in all of the simulations. Using TE polarization, no particle plasmon resonance is induced so that the coupled mode may be attributed solely to the interaction between the waveguide and the FP resonance modes. In the simulation, we employed the complex refractive index of silver given by the GSOLVER software.

Figure 3(a) shows the simulated transmission spectra of the waveguide FP microcavity arrays with the grating period changed from 350 to $500 \mathrm{~nm}$. Other parameters of the virtual device are given in the caption of Fig. 3(a). Clearly, the
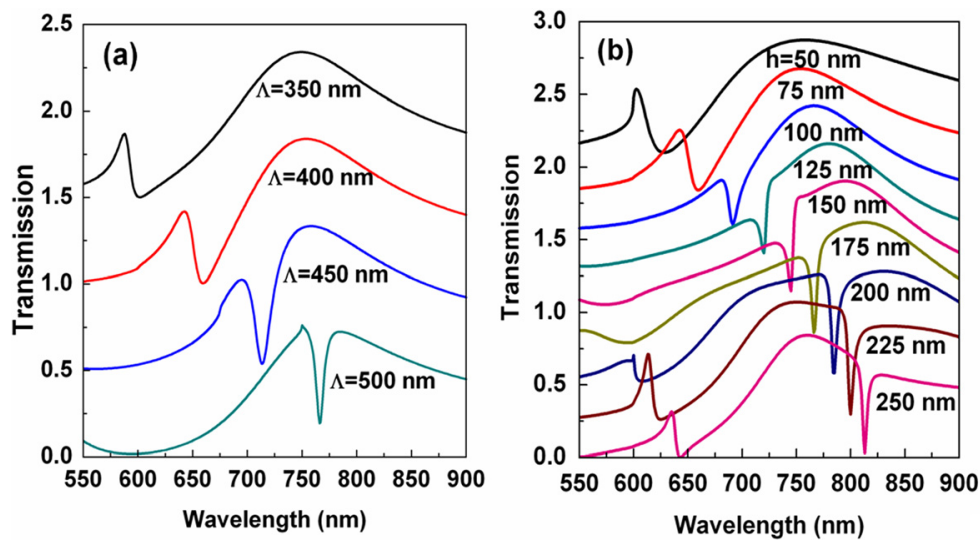

FIG. 3. (Color online) Transmission spectra of the waveguide FP microcavity array. (a) The grating period is changed from 350 to $500 \mathrm{~nm}$ in steps of $50 \mathrm{~nm}$. Other parameters used: $f=0.5, t=25$ $\mathrm{nm}, d=125 \mathrm{~nm}, h=75 \mathrm{~nm}$, and $n_{\text {sub }}=1.5$. (b) The waveguide thickness is changed from $50 \mathrm{~nm}$ to $250 \mathrm{~nm}$ in steps of $25 \mathrm{~nm}$ with the grating period fixed at $\Lambda=400 \mathrm{~nm}$. Other parameters used: $f=0.5, t=25 \mathrm{~nm}, d=125 \mathrm{~nm}$, and $n_{\text {sub }}=1.5$. 

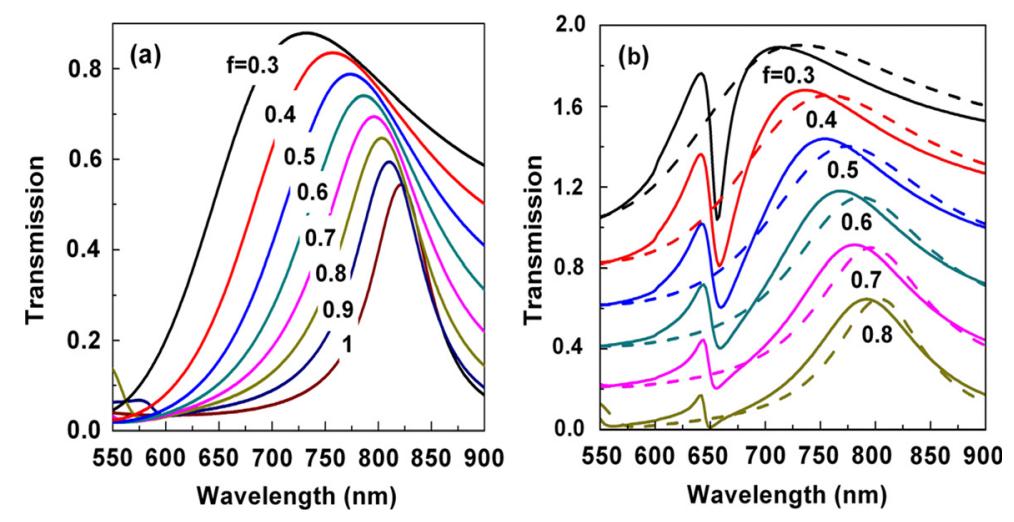

FIG. 4. (Color online) (a) Transmission spectra of non-waveguided FP microcavity array with the duty cycle changed from $f=0.3$ to 1 in steps of 0.1 . Other parameters used: $\Lambda=400 \mathrm{~nm}$, $t=25 \mathrm{~nm}, d=125 \mathrm{~nm}$. (b) Transmission spectra of waveguide FP microcavity array with the duty cycle changed from $f=0.3$ to 0.8 in steps of 0.1 (solid curves). Other parameters used: $\Lambda=400$ $\mathrm{nm}, t=25 \mathrm{~nm}, d=125 \mathrm{~nm}, h=75 \mathrm{~nm}$, and $n_{\text {sub }}=1.5$. As a comparison, the corresponding transmission spectra (dashed curves) of the non-waveguide FP microcavities located on $\mathrm{ZnS}$ substrate are also shown in (b).

waveguide resonance mode is tuned from 587 to $766 \mathrm{~nm}$ and the FP pass band extends from about 600 to longer than 900 $\mathrm{nm}$. The character of the waveguide resonance mode changes when crossing from the FP stop into the pass band. A clear asymmetric transmission peak can be observed at about 587 $\mathrm{nm}$ for a grating period of $350 \mathrm{~nm}$ and waveguide resonance overlaps with the stop band of the FP microcavity, whereas a clear almost symmetric dip in the transmission spectrum at about $766 \mathrm{~nm}$ is produced for a grating period of 450 or 500 $\mathrm{nm}$ and the waveguide resonance mode strongly overlaps with the FP pass band. The transition between the peak and dip characters can be observed for a grating period of 400 $\mathrm{nm}$. The evolution of the resonance lineshape of the waveguide mode is in concordance with the Fano picture.

Furthermore, the spectral position of the waveguide resonance mode can also be tuned by changing the thickness of the waveguide layer, as shown in Fig. 3(b). When the thickness of the waveguide is increased from 50 to $250 \mathrm{~nm}$, the waveguide resonance mode changes from a clear transmission peak at about $603 \mathrm{~nm}$ to a clear dip at $813 \mathrm{~nm}$ in the transmission spectrum, other parameters of the virtual device are given in the caption of Fig. 3(b). It can also be seen clearly in Fig. 3(b) that for waveguide layers thicker than $200 \mathrm{~nm}$, a higher order process can be observed giving rise to an additional sharp transmission peak in the range of the FP stop band. This peak shifts from about 600 to $635 \mathrm{~nm}$ and also shows the asymmetry typical for a Fano resonance when the waveguide thickness is tuned from 200 to $250 \mathrm{~nm}$.

Both the bandwidth and the spectral position of the FP pass or stop band can be tuned by changing the duty cycle of the FP microcavity arrays. Fig. 4(a) shows the calculated transmission spectra of the non-waveguide FP microcavity arrays as a function of duty cycle, where the material of the substrate is also chosen to be $\mathrm{ZnS}$ and the duty cycle is changed from 0.3 to 1 . Other fixed parameters of the virtual device are given in the caption of Fig. 4(a). The center wavelength of the pass band of the FP microcavities shifts from 728 to $821 \mathrm{~nm}$ and the bandwidth at full width at half maximum (FWHM) is reduced from about 260 to $65 \mathrm{~nm}$. The duty cycle of 1 corresponds to a single FP cavity of infinite width. Fig. 4(b) depicts the transmission spectra of FP microcavity arrays of different duty cycles with (solid curves) and without (dashed curves) coupling to a $\mathrm{ZnS}$ waveguide. It is clear that the coupling to the waveguide yields the sharp features of a waveguide resonance mode. Again its character and corresponding Fano-parameter $q$, i.e., as a narrow symmetric resonance $(q=0)$, an asymmetric resonance (finite $q$ ) or an antiresonance $(q=\infty)$ depends on its spectral position, i.e., within the FP stop or pass band. When the grating period is fixed at $400 \mathrm{~nm}$ and waveguide thickness at $75 \mathrm{~nm}$, the waveguide resonance mode is located at about $650 \mathrm{~nm}$. As the duty cycle of the grating is increased from 0.3 to 0.8 , the pass band shifts from 712 to $789 \mathrm{~nm}$ and the bandwidth at FWHM is reduced from 267 to $119 \mathrm{~nm}$. Thus, the waveguide resonance mode is observed as a transmission dip for $f=0.3$ and a transmission peak for $f=0.8$. This behavior confirms convincingly the coupling effects between the waveguide and FP resonance modes.

In conclusion, the Fano-like coupling between a narrowband waveguide resonance mode and a broad-band or quasicontinuous Fabry-Pérot resonance mode is investigated theoretically in a nanodevice consisting of periodically arranged Fabry-Pérot microcavities on the top of a waveguide layer. The understanding of these effects is potentially important for a better design of band-selective filters, polarizers, and optical switches with high tunability.

The authors acknowledge the National Natural Science Foundation of China (11074018), the Program for New Century Excellent Talents in University (NCET), and the Research Fund for the Doctoral Program of Higher Education of China (RFDP, 20091103110012) for financial support.

\footnotetext{
${ }^{1}$ A. Sharon, D. Rosenblatt, and A. A. Friesem, J. Opt. Soc. Am. A 14, 2985 (1997).

${ }^{2}$ X. P. Zhang, S. F. Feng, H. M. Liu, and L. Wang, Appl. Phys. Lett. 93, 093113 (2008).

${ }^{3}$ X. P. Zhang, H. M. Liu, J. R. Tian, Y. R. Song, and L. Wang, Nano Lett. 8, 2653 (2008).

${ }^{4}$ X. P. Zhang, B. Q. Sun, J. M. Hodgkiss, and R. H. Friend, Adv. Mater. 20, 4455 (2008)

${ }^{5}$ A. Christ, S. G. Tikhodeev, N. A. Gippius, J. Kuhl, and H. Giessen, Phys. Rev. Lett. 91, 1839011 (2003).

${ }^{6}$ B. Lukyanchuk, N. I. Zheludev, S. A. Maier, N. J. Halas, P. Nordlander, H. Giessen, and C. T. Chong, Nature Mater. 9, 707 (2010).

${ }^{7}$ N. Liu, T. Weiss, M. Mesch, L. Langguth, U. Eigenthaler, M. Hirscher, C. Sonnichsen, and H. Giessen, Nano Lett. 10, 1103 (2010).

${ }^{8}$ S. Yano, R. Schroeder, H. Sakai, and B. Ullrich, Appl. Phys. Lett. 82, 2026 (2003).

${ }^{9}$ S. F. Feng, X. P. Zhang, J. Y. Song, H. M. Liu, and Y. R. Song, Opt. Express 17, 426 (2009).

${ }^{10}$ U. Fano, Phys. Rev. 124, 1866 (1961).

${ }^{11}$ See http://www.gsolver.com for information about the GSOLVER software for the full-vector solution of periodic structures.

${ }^{12}$ M. G. Moharam and T. K. Gaylord, J. Opt. Soc. Am. 71, 811 (1981).
} 